\title{
Correlation of Modic Changes with Sagittal Lumbopelvic Parameters
}

\author{
Xiaoping $\mathrm{Mu}$ (D) $^{\prime}$ \\ Chengqiang Yu' \\ Seong Woong $\mathrm{Kim}^{2}$ \\ Yufu Ou' \\ Jianxun Wei' \\ Karsten Schöller ${ }^{3}$ \\ 'Department of Spine Surgery, The \\ People's Hospital of Guangxi Zhuang \\ Autonomous Region, Guangxi Academy \\ of Medical Sciences, Nanning, Guangxi, \\ People's Republic of China; ${ }^{2}$ Department \\ of Neurosurgery, Justus-Liebig-University, \\ Giessen, Hessen, Germany; ${ }^{3}$ Clinic for \\ Spinal Surgery, Schoen Clinic Hamburg \\ Eilbek, Hamburg, Germany
}

Purpose: The main aim of this retrospective study was to analyze lumbopelvic sagittal parameters among the three different types of Modic changes (MCs). Furthermore, correlations between the sizes of MCs and the number of involved lumbar levels with lumbopelvic parameters are investigated.

Methods: A total of 263 adult subjects with MCs at a single institution from September 2015 to October 2020 who underwent lumbar x-ray examinations and magnetic resonance imaging were included in this retrospective study. Types of MCs, sizes of MCs, lumbar levels involved by MCs as well as lumbopelvic sagittal parameters from each subject were evaluated by two authors.

Results: Lumbar lordosis (LL), sacral slope (SS), and pelvic incidence (PI) in subjects with MC grade 1 were significantly smaller than in those with MC grade 2 and grade $3(p<0.05)$. Lumbopelvic sagittal parameters decreased significantly as the sizes aggravated $(p<0.01)$. Triple lumbar levels with MCs showed a significant increase in PI-LL $(\mathrm{p}<0.05)$ and decrease in LL $(p<0.01)$, SS $(p<0.01)$, and PI $(p<0.01)$ when compared to MCs at single and double lumbar levels.

Conclusion: MC grade 1, severe MCs, and lumbar multi-segmental MCs were significantly linked to lumbar sagittal imbalance.

Keywords: Modic changes, sagittal balance, lumbopelvic parameter, pelvic incidence, lumbar lordosis, sacral slope

\section{Introduction}

Chronic low back pain (CLBP) is the most common symptom of patients with degenerative disc disease who are seeking professional treatment. ${ }^{1}$ Considering its essential impact on the individual, communities, and health care systems, ${ }^{1}$ much more attention should be paid to it. As a potential source for CLBP, ${ }^{2-5}$ abnormal signal changes in the subchondral bone marrow adjacent to the endplates, also known as Modic changes (MCs), were first discussed by Modic et al ${ }^{6,7}$ in 1988.

In the decades since the preliminary elaboration of MCs by Modic et al, there has been an ongoing dispute about their pathogenesis. Biomechanical injury, inflammatory, and low-grade bacterial infection are generally considered to be the main causes of the development of MCs. ${ }^{3,8,9}$ These 3 models may not be independent of each other but are closely interrelated, each of them potentially triggering a cascade of events leading to the development of MCs. ${ }^{8}$ However, biomechanical injury of the endplates can provide good anatomical conditions for the "cross-talk" of pro-inflammatory mediators between the disc and subchondral bone marrow as well the colonization and spread of bacteria, which may be one of the important
Correspondence: Xiaoping Mu; Jianxun

Wei

Email muxbin1991@hotmail.com;

jxweil972@I63.com 
prerequisites for the development of MCs. ${ }^{10}$ The increased shear force acting on the degenerative disc would cause the micro-fracture of the vertebral endplates, ${ }^{11,12}$ showing bone marrow edema related to $\mathrm{MC1}$.

The sagittal spinopelvic contour plays an important role in the normal function of the spine and in various diseased states. ${ }^{13}$ Knowledge of its normal composed elements would be crucial for understanding its significance in body balance and alignment. ${ }^{14}$ Once abnormal, the body needs to expend more energy to compensate for the abnormal sagittal alignment for balance. ${ }^{15}$ Recently, several studies $^{10,13,16}$ have investigated sagittal parameters among patients with and without MCs. The strong relationships between MCs and spinal sagittal imbalance might potentially provide new insight into the mechanism of MCs leading to CLBP.

However, previous studies did not investigate the potential effects of the sizes of MCs and the number of lumbar levels affected by MCs on lumbar sagittal parameters. Therefore, the purpose of this study was to analyze lumbopelvic sagittal parameters in correlation to $\mathrm{MC}$ grades, sizes, and number of involved lumbar levels.

\section{Materials and Methods}

This study is a part of the research program"the imaging study of the intervertebral disc and endplate degeneration for patients with cervical/lumbar degenerative diseases"approved by our Institutional Ethical Committee in 2016. We conceived and implemented this study in April 2021.

\section{Study Population}

According to the aims of this study, the inclusion and exclusion criteria were formulated for this study before selecting the appropriate subjects. The subjects who met the following criteria were included in this study: (1) Age over 18 years on admission; (2) Subjects underwent lumbar X-ray examination in the standing position and lumbar magnetic resonance imaging (MRI) scanning in the supine position from September 2015 to October 2020; (3) There was evidence of the imaging appearance of one of three types of MCs occurring at any lumbar level on MRI; (4) Radiological examinations complied with the standard operating procedures and the images were of high quality. The subjects were excluded if they had a prior history of lumbar surgery, significant spinal deformities (spinal scoliosis $\geq 15^{\circ}$ ), spinal tumor, spinal trauma, spinal infections or inflammations, or abnormal postures caused by lower extremity diseases.

Finally, 263 adult subjects who met the abovementioned criteria were included in this retrospective study. All images included in this study were coded and covered subjects' personal information. The study protocol was approved by the ethics committee of The People's Hospital of Guangxi Zhuang Autonomous Region (No. 2016-12) and we implemented this study in strict accordance with the principles of the Declaration of Helsinki. Patients who participated in this study signed an informed consent form with knowledge of the study's purposes and relevant details.

\section{Imaging Evaluation and Measurement}

All subjects were examined by lumbar MRI (supine position) and X-ray (standing position). The scanning area was included at least L1 to the femoral heads. Subjects' images were recorded in the electronic medical system of our hospital.

\section{MRI Protocol}

All included patients were examined with $3.0 \mathrm{~T}$ magnetic resonance units (Siemens, Germany) with the following parameters: (1) the sagittal T1-weighted images (T1WI): time to echo (TE): $8 \mathrm{~ms}$, time of repetition (TR): $400 \mathrm{~ms}$, slice thickness (ST): $4 \mathrm{~mm}$, and field of view (FOV): $512 * 512$ pixel; (2) the sagittal T2-weighted images (T2WI): TE: $100 \mathrm{~ms}$, TR: $3000 \mathrm{~ms}$, ST: $4 \mathrm{~mm}$, FOV: $512 * 512$ pixel.

\section{Assessment of Three Types of MCs}

Two spinal surgeons (X.P.M and C.Q.Y) with more than 3 -year experience were allocated to independently evaluate all MR images for identifying the type of MCs. Three types of MCs (Figure 1) proposed by Modic et $\mathrm{al}^{6,7}$ were defined by their appearances on both TIWI and T2WI. Modic type 1 change (MC1) indicates a hypointense signal on T1WI and a hyperintense signal on T2WI. Modic type 2 change (MC2) reflects the hyperintense signals both on T1- and T2WIs. Whereas, Modic type 3 change (MC3) is characterized by the hypointense signals both on $\mathrm{T} 1-$ and T2WIs.

\section{Parameters Measurement}

The above-mentioned 2 reviewers independently measured all radiological parameters on X-ray images from 15 randomly selected subjects and calculated the inter-observer reliability thereafter. In addition, the measurement of these 

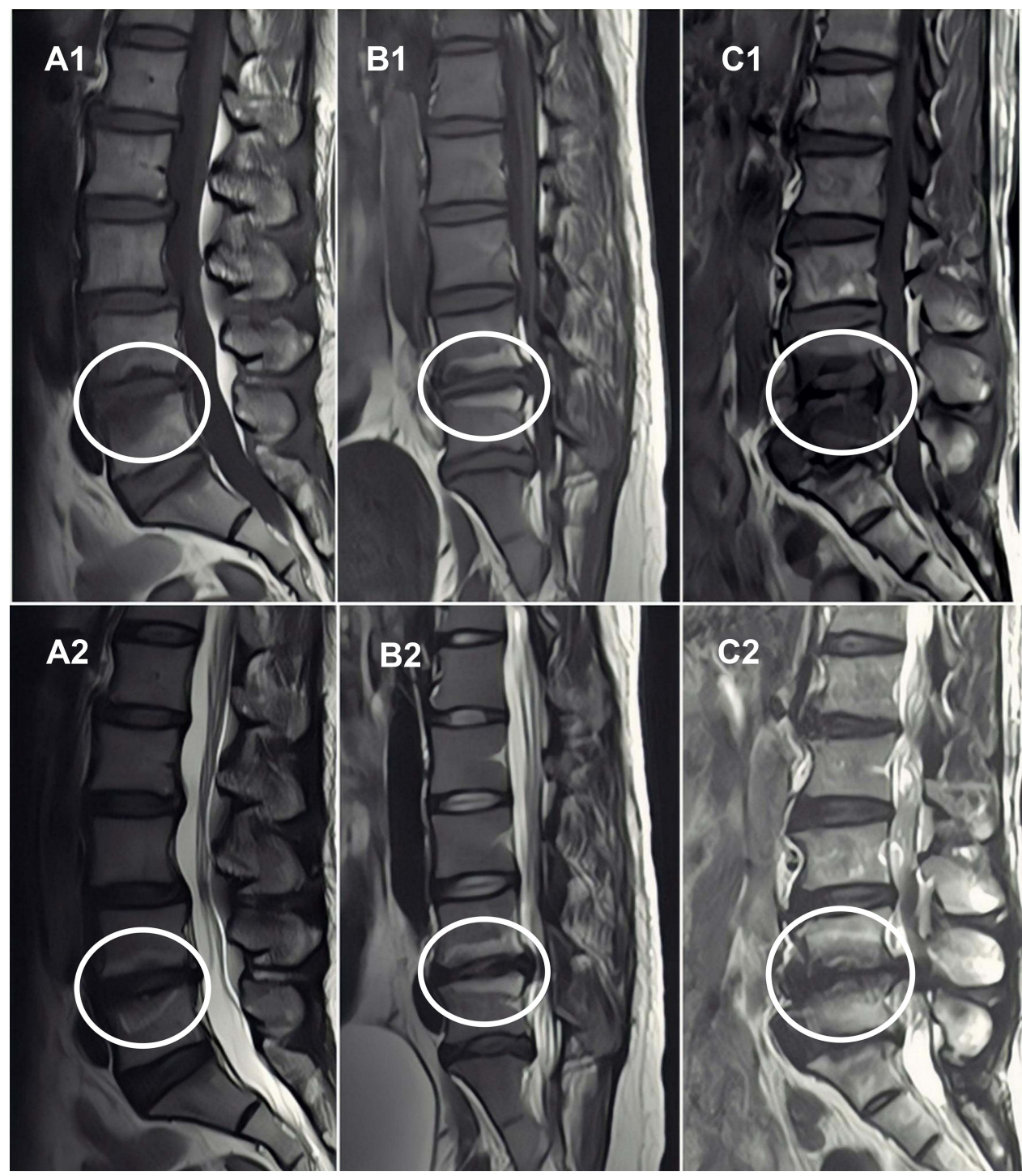

Figure I The imaging presentations of the three types of Modic changes (MCs) on magnetic resonance imaging. Modic type I change: decreased signal intensity on TIweighted images (TIWIs, (AI)) and increased signal intensity on T2-weighted images (T2Wls, (A2)); MC2: increased signal intensity both on TIWls (BI) and T2Wls (B2); MC3: decreased signal intensity both on TIWls (CI) and T2Wls (C2).

parameters were repeated 10 days after the first measurement and the intra-observer reliability was calculated.

Surgimap $^{\circledR}$ 2.3.2.1 software (Nemaris Inc., New York, USA) was used to measure the parameters on X-ray images. The sagittal parameters were measured in the neutral position on X-ray images (Figure 2). Lumbar lordosis (LL) was the angle between by two lines which were parallel to the superior endplate of $\mathrm{L} 1$ and the inferior endplate of L5. The conformation of sacral slope (SS) was similar to LL, but it consisted of one line parallel to the superior endplate of S1 and the horizontal line. Pelvic tilt (PT) was an angle formed by a line through the midpoint of the S1 superior endplate and the center of the femoral head and a vertical line through the center of the femoral head. Pelvic incidence (PI), which is an angle that based on a line perpendicular to the sacral endplate and a line through the midpoint of the S1 superior endplate and the center of the femoral head, is a fixed value determined by the sum of the SS and PT.

\section{Size of MCs}

We adopted a classification proposed by Hanımoğlu et $\mathrm{al}^{2}$ to categorize the distribution of three types of MCs at the endplate and vertebral bodies. This classification method allowed us to divide the vertebrae into 4 equal parts including 16 small units across the horizontal and vertical 


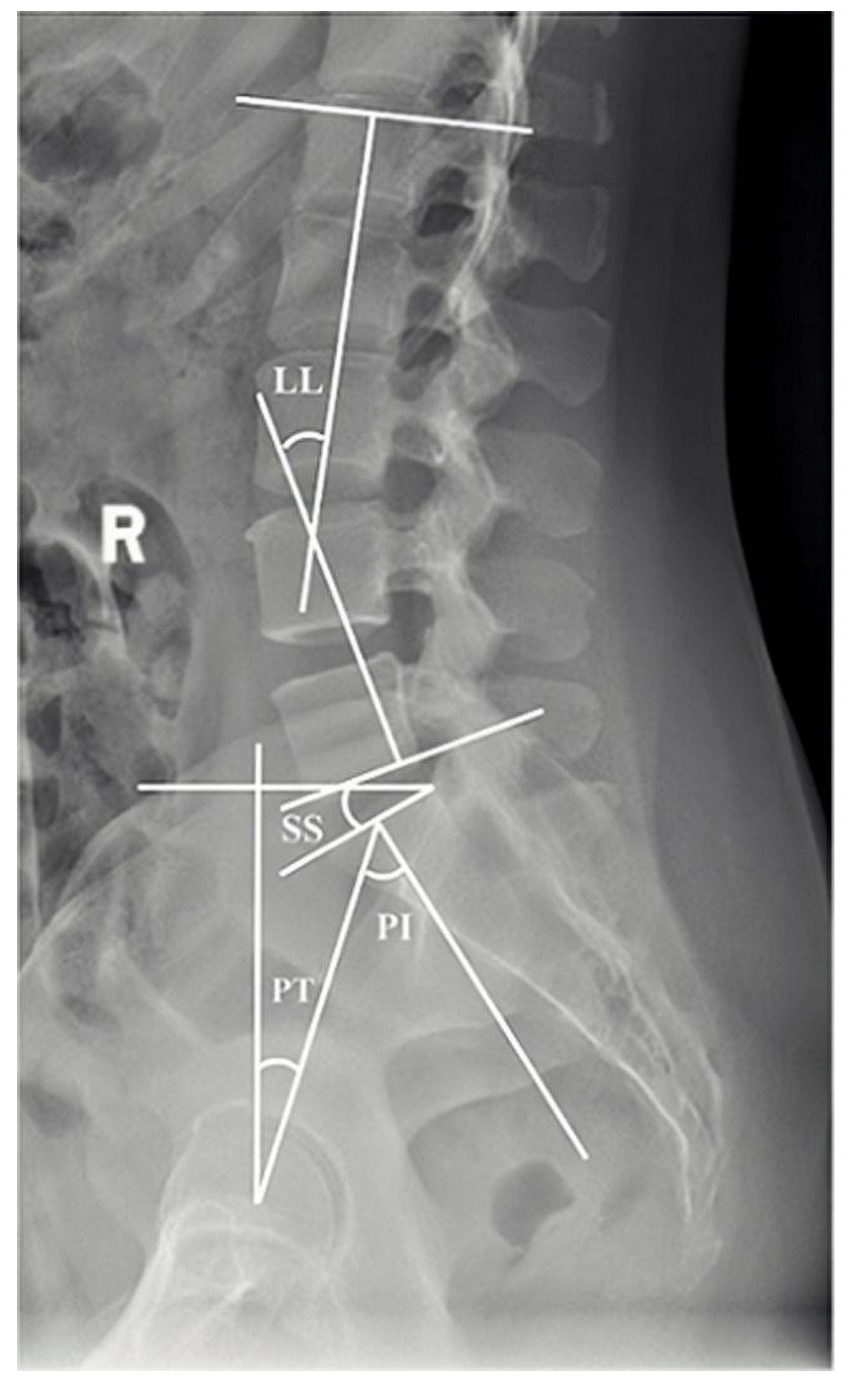

Figure 2 Measurement of sagittal spinopelvic parameters in the lumbar spine. Abbreviations: LL, lumbar lordosis; SS, sacral slope; PI, pelvic incidence; PT, pelvic tilt.

planes (Figure 3). The total sizes of MCs were calculated by summing each small unit of MCs involvement. Therefore, we could determine the involvement area of MCs as from 0 to IV4 (Table 1). The involvement area of MCs with less than 1/4 of the vertebral body horizontally or vertically was defined as slight, larger than $1 / 4$ but smaller than $3 / 4$ of the vertebral body as moderate, and more than $3 / 4$ of the vertebral body as severe.

\section{Statistical Analysis}

IBM SPSS 22.0 (SPSS Inc., Armonk, New York) was employed to perform statistical analysis. We used mean \pm standard deviation (SD) to describe continuous variables. Two independent samples $t$-test or one-way variance analysis was applied to analyze them if these continuous



Figure 3 Evaluation the sizes of Modic changes in the horizontal and vertical planes.

variables complied with the normal distribution. Otherwise, the Mann-Whitney $U$-test or Kruskal-Wallis test was used. Categorical variables were expressed as percentages (\%) and numbers. A $p$-value with two-tail less than or equal to 0.05 was considered as a significant difference. We used the intra-class correlation coefficient (ICC) to evaluate the inter-and intra-observer reliability. ${ }^{17}$ The ICC value of inter-and intra-observer reliability for types of MCs and radiological parameters were all more than 0.8 and were graded as good to excellent.

\section{Results}

\section{Baseline Characteristics}

A total of 263 subjects were included in this retrospective study, with a mean age of 57 (SD: 9.9) years and a mean body mass index (BMI) of $22.7 \mathrm{~kg} / \mathrm{m}^{2}$ (SD: 3.0). There were 150 females (57\%) and 113 males (43\%). The incidence of MC1, MC2, and MC3 among the included population was 30\% (79/263), 46\% (121/263), and 24\% (63/ 263), respectively. According to the sizes of MCs, 113 subjects were defined as slight, 82 subjects as moderate, and the remaining 68 subjects as severe. In the present work, we did not identify any MCs occurring at the segment of L1/2 or L2/3. Over $70 \%$ of MCs (189/263) were observed at a single lumbar level, of which $27 \mathrm{MCs}$ (14.3\%) occurred at L3/4, $64(33.9 \%)$ at L4/5, and 98 $(51.8 \%)$ at $\mathrm{L} 5 / \mathrm{S} 1$. The incidence of MCs at double and 
Table I The Grading System Regarding the Involvement Area of Modic Changes (MCs)

\begin{tabular}{|l|c|c|c|c|c|}
\hline \multicolumn{2}{|l|}{ Groups } & \multicolumn{4}{|c|}{ Involvement Area of MCs at the Horizontal Plane } \\
\cline { 2 - 6 } & I Small unit & 2 Small units & 3 Small units & 4 Small units \\
\hline \multirow{3}{*}{ Involvement Area of MCs at the Vertical Plane } & I Small unit & II & I2 & I3 & I4 \\
\cline { 2 - 7 } & 2 Small units & III & II2 & II3 III3 & III4 \\
\cline { 2 - 7 } & 3 Small units & IIII & III2 & IV3 & IV4 \\
\cline { 2 - 7 } & 4 Small units & IVI & IV2 & &
\end{tabular}

Note: Red: slight; blue: moderate; brown: severe.

Table 2 Baseline Characteristics of the Included Subjects

\begin{tabular}{|c|c|}
\hline Number of Patients & $n=263$ \\
\hline Gender & male: II3 female: 150 \\
\hline Age (years)* & $57.0 \pm 9.9$ \\
\hline Body mass index $\left(\mathrm{kg} / \mathrm{m}^{2}\right)^{*}$ & $22.7 \pm 3.0$ \\
\hline Types of Modic changes (MCs) & $\begin{array}{l}\text { Modic type I change: } 79(30.0 \%) \\
\text { Modic type } 2 \text { change: } 12 \text { I ( } 46.0 \%) \\
\text { Modic type } 3 \text { change: } 63(24.0 \%)\end{array}$ \\
\hline Extents of MCs & $\begin{array}{l}\text { Slight: } 113(43.0 \%) \\
\text { Moderate: } 82(31.2 \%) \\
\text { Severe: } 68 \text { (25.8\%) }\end{array}$ \\
\hline Lumbar levels with MCs & $\begin{array}{l}\text { Single level: I89 (7I.9\%) } \\
27 \text { (I4.3\%, L3/4) } \\
64 \text { (33.9\%, L4/5) } \\
98 \text { (5I.8\%, L5/SI) } \\
\text { Double levels: } 46 \text { (I7.5\%) } \\
18 \text { (39.1\%, L3-5) } \\
28 \text { (60.9\%, L4-SI) } \\
\text { Triple levels: } 28 \text { (I0.6\%, L3-SI) }\end{array}$ \\
\hline
\end{tabular}

Note: *Mean $\pm S D$.

triple lumbar levels was $17.5 \%$ (46/263; L3-5: 18; L4-S1: 28 ) and $10.7 \%$ (28/263, L3-S1). Baseline characteristics of the included subjects are shown in Table 2.

\section{Lumbar Sagittal Parameters}

The results of lumbar sagittal parameters among the three types of MCs are shown in Table 3. The statistical analysis revealed that several lumbar sagittal parameters in subjects with MC1 (LL: $-25.0 \pm 9.6$; SS: $22.3 \pm 7.7$; and PI: 39.8 $\pm 7.4)$ were significantly smaller than the groups of MC2 (LL: $-29.0 \pm 10.5$; SS: $26.4 \pm 8.5$; and PI: 43.9 \pm 7.6 ) and MC3 (LL: $-29.1 \pm 9.8$; SS: $26.7 \pm 7.8$; and PI: 43.5 \pm 6.5 ) $(p<0.05)$, However, we did not detect any statistical differences in PT and PI-LL between MC1 (PT:17.4 \pm 4.0 ; PI-LL

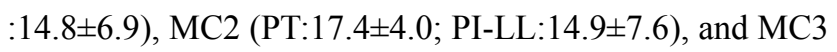
(PT:16.8 \pm 3.8 ; PI-LL:14.3 \pm 6.5$)(\mathrm{p}>0.05)$.

263 subjects were allocated to three groups (slight, moderate, and severe) based on the sizes of MCs (Table 4). The subjects with severe MCs (LL: $-21.5 \pm 7.9$; SS: 19.9 \pm 7.0 ; and PI: $37.0 \pm 6.2$ ) were significant smaller LL, SS, and PI values than those of slight (LL: -32.6 \pm 10.4 ; SS: $29.9 \pm 7.7$; and PI: $46.7 \pm 6.6$ ) or moderate MCs (LL: $-26.4 \pm 8.3$; SS: 23.3 \pm 6.5 ; and PI: $41.4 \pm 6.1$ ), with statistical differences among the three groups $(p<0.01)$. However, no significant differences in PT and PI-LL were detected between the 3 groups ( $p>0.05$ ).

Considering that the potential influence of lumbar levels involved by MCs on lumbar sagittal parameters,

Table 3 Lumbar Sagittal Parameters Among Three Types of Modic Changes (MCs)

\begin{tabular}{|c|c|c|c|c|c|}
\hline \multicolumn{2}{|l|}{$\begin{array}{l}\text { Types } \\
\text { Characteristics }\end{array}$} & $\begin{array}{c}M C I \\
(n=79)\end{array}$ & $\begin{array}{c}M C 2 \\
(n=12 I)\end{array}$ & $\begin{array}{c}M C 3 \\
(n=63)\end{array}$ & $p$ \\
\hline \multirow[t]{5}{*}{ Sagittal parameters } & Lumbar lordosis (LL, degrees) & $-25.0 \pm 9.6 *$ & $-29.0 \pm 10.5$ & $-29.1 \pm 9.8$ & $<0.05$ \\
\hline & Sacral slope (degrees) & $22.3 \pm 7.7^{*}$ & $26.4 \pm 8.5$ & $26.7 \pm 7.8$ & $<0.01$ \\
\hline & Pelvic tilt (degrees) & $17.4 \pm 4.0$ & $17.4 \pm 4.0$ & $16.8 \pm 3.8$ & n.s. \\
\hline & Pelvic incidence (PI, degrees) & $39.8 \pm 7.4^{*}$ & $43.9 \pm 7.6$ & $43.5 \pm 6.5$ & $<0.01$ \\
\hline & PI-LL (degrees) & $14.8 \pm 6.9$ & $14.9 \pm 7.6$ & $14.3 \pm 6.5$ & n.s. \\
\hline
\end{tabular}

Notes: mean \pm SD; *Compared with $M C 2$ and MC3, P<0.05.

Abbreviation: n.s., no significance. 
Table 4 Lumbar Sagittal Parameters in Subjects with Different Involvement Extents of Modic Changes

\begin{tabular}{|c|c|c|c|c|c|}
\hline $\begin{array}{l}\text { Extents } \\
\text { Characteristics }\end{array}$ & & $\begin{array}{c}\text { Slight } \\
(n=1 \mid 3)\end{array}$ & $\begin{array}{l}\text { Moderate } \\
(n=82)\end{array}$ & $\begin{array}{l}\text { Severe } \\
(n=68)\end{array}$ & $p$ \\
\hline Sagittal parameters & $\begin{array}{c}\text { Lumbar lordosis (LL, degrees) } \\
\text { Sacral slope (degrees) } \\
\text { Pelvic tilt (degrees) } \\
\text { Pelvic incidence (PI, degrees) } \\
\text { PI-LL (degrees) }\end{array}$ & $\begin{array}{c}-32.6 \pm 10.4^{*} \\
29.9 \pm 7.7^{*} \\
16.8 \pm 3.8 \\
46.7 \pm 6.6^{*} \\
14.1 \pm 7.7\end{array}$ & $\begin{array}{c}-26.4 \pm 8.3^{*} \\
23.3 \pm 6.5^{*} \\
18.1 \pm 3.8 \\
41.4 \pm 6.1^{*} \\
15.0 \pm 6.7\end{array}$ & $\begin{array}{c}-21.5 \pm 7.9 \\
19.9 \pm 7.0 \\
17.1 \pm 4.1 \\
37.0 \pm 6.2 \\
15.4 \pm 6.7\end{array}$ & $\begin{array}{c}<0.01 \\
<0.01 \\
\text { n.s } \\
<0.01 \\
\text { n.s. }\end{array}$ \\
\hline
\end{tabular}

Notes: mean $\pm S D$; *Compared with group of severe, $\mathrm{P}<0.05$.

Abbreviation: n.s., no significance.

Table 5 Lumbar Sagittal Parameters in Subjects with Different Lumbar Levels Involved by Modic Changes

\begin{tabular}{|c|c|c|c|c|c|}
\hline \multicolumn{2}{|c|}{$\begin{array}{l}\text { Lumbar Segments } \\
\text { Characteristic }\end{array}$} & \multirow{2}{*}{$\begin{array}{c}\begin{array}{c}\text { Single Level } \\
(n=189)\end{array} \\
-28.9 \pm 10.5\end{array}$} & \multirow{2}{*}{$\begin{array}{c}\begin{array}{c}\text { Double Levels } \\
(n=50)\end{array} \\
-28.3 \pm 8.3\end{array}$} & \multirow{2}{*}{$\begin{array}{c}\begin{array}{c}\text { Triple Levels } \\
(n=24)\end{array} \\
-18.5 \pm 6.3^{*}\end{array}$} & \multirow{2}{*}{$\begin{array}{c}\boldsymbol{p} \\
<0.01\end{array}$} \\
\hline Spinopelvic & Lumbar lordosis (LL, degrees) & & & & \\
\hline parameters & Sacral slope (degrees) & $26.0 \pm 3.9$ & $25.5 \pm 7.6$ & $18.6 \pm 5.3^{*}$ & $<0.01$ \\
\hline & Pelvic tilt (degrees) & $17.3 \pm 3.9$ & $16.5 \pm 3.7$ & $18.3 \pm 4.1$ & n.s \\
\hline & Pelvic incidence (PI, degrees) & $43.4 \pm 7.6$ & $42.0 \pm 6.6$ & $36.9 \pm 5.6^{*}$ & $<0.01$ \\
\hline & PI-LL (degrees) & $14.5 \pm 7.3$ & $13.7 \pm 6.1$ & $18.5 \pm 6.5^{\#}$ & $<0.05$ \\
\hline
\end{tabular}

Notes: mean \pm SD; *Compared with the groups of single and double levels, $\mathrm{P}<0.01$. ${ }^{*}$ Compared with the group of double levels, $\mathrm{P}<0.05$.

Abbreviation: n.s., no significance.

the included 263 subjects were divided into 3 groups: single lumbar level with MCs, double lumbar levels with MCs, and triple lumbar levels with MCs (Table 5). The analysis showed that triple lumbar levels with MCs had a significant decrease in LL (-18.5 \pm 6.3$)$, SS (18.6 \pm 5.3$)$, and PI $(36.9 \pm 5.6)(\mathrm{p}<0.01)$ and a significant increase in PILL $(18.5 \pm 6.5, \mathrm{p}<0.05)$ when compared to those MCs at single (LL: $-28.9 \pm 10.5$; SS: $26.0 \pm 3.9$; PI: $43.4 \pm 7.6$; and PI-LL: $14.5 \pm 7.3)$ and double lumbar levels (LL: -28.3

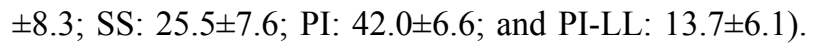

\section{Discussion}

Previous studies ${ }^{13,16}$ have reported that lumbar sagittal parameters are generally related to the development of MCs, whereas the correlation of the MC type, the size, and the levels involved with lumbopelvic parameters is not well understood. The results of this study showed that LL, SS, and PI are closely linked to $\mathrm{MC1}$ and negatively correlated to the size of MCs. Despite MCs mainly involving one lumbar level, triple lumbar levels with MCs tend to influence sagittal balance to a major extent.

Radiographic parameters including LL, SS, PT, PI, and PI-LL are the most commonly used to evaluate the sagittal spinopelvic alignment in clinical practice and studies. LL and SS reflect the overall balance of the lumbar spine.
A loss of LL or SS may be strongly associated with the development of CLBP by disrupting the local balance of the lumbar spine. ${ }^{18,19}$ However, in response to the changes of LL and SS, subjects could change their PT via the movement of a hip joint to maintain normal sagittal balance. ${ }^{20}$ Therefore, PT can be thought of as a compensatory mechanism for sagittal imbalance and spinal deformity. ${ }^{21} \mathrm{PI}$, a well-known pelvic morphological parameter, always maintains a constant value at the end of growth and is significantly associated with other radiographic parameters such as LL, $\mathrm{SS}$, and $\mathrm{PT}^{22,23}$ However, since PI is constant, a mismatch between PI and LL may be caused by a loss of LL, thus affecting the global sagittal balance. A PI-LL of 10 degrees or less is usually considered the ideal sagittal matching between the lumbar spine and the pelvis. ${ }^{24}$

The relationship between MCs and the sagittal parameters is currently controversial and is considered to be interactive. The damage to the endplate generally precedes the intervertebral disc: the calcification and lesion of the endplate would decrease its permeability, and therefore, impede the nutritional supply of the disc to a certain extent, which accelerates degenerative changes of the intervertebral disc. ${ }^{25,26}$ Additionally, the degeneration of the vertebral endplates causes the tilts of the upper and 
lower edges, the superior and inferior edge of the vertebral body, and the inconsistency of the anterior and posterior intervertebral space, ${ }^{27}$ resulting in the instability of the adjacent vertebral body and the changing of the sagittal balance.

Due to the unique anatomy of the lumbar spine, it is subjected to relatively high mechanical stress. ${ }^{28}$ In the process of degeneration including the endplate calcification, a slight change in the sagittal parameters can be anticipated and compensated by the local postural adjustment. However, the localized postural adjustment may cause mechanical compensatory changes in the whole spine. Therefore, LL, SS, and PI have to be changed to accommodate this mechanical compensatory mechanism over time. $^{29}$

Previous studies ${ }^{13,16,30-32}$ have shown strong relationships between MCs and sagittal spinopelvic parameters. The lower LL and SS are more often seen in subjects with lumbar degenerative disease. Recent studies ${ }^{13,16}$ have demonstrated that LL and SS were significantly decreased in subjects with MCs compared to those without MCs. However, the relationship between MCs and PI is not well-understood, as the results of existing studies on this topic vary greatly. ${ }^{13,22}$

In the present work, we performed a detailed stratification analysis based on the type of MCs, the sizes, and lumbar segments involved by MCs. The results indicated that subjects with $\mathrm{MC1}$, severe $\mathrm{MCs}$, and triple lumbar levels with MCs had significantly smaller LL as well as SS. As mentioned before, biomechanical injury to the vertebral endplates plays an important role in the development of MCs. We speculate that the lower values of the LL and SS not only reduce the resistant capacity of the intervertebral disc for the axial loading but also increase the axial forces on the vertebral endplates, leading to the endplates microfractures, which may be able to respond to why the lower LL and SS cause MCs. However, their processes might not be disparate but are interactive. Rapid progression of degenerative changes in the disc with MCs and the loss of disc height ${ }^{33}$ may both contribute to the lower LL. ${ }^{21}$ A loss of LL has the potential to lead to the anterior translation of the gravitational axis. The value of SS is passively decreased to maintain the normal gravitational axis in the compensation process. ${ }^{22}$

A lower PI significantly alters the transmission of mechanical load in the spine and imposes excessive axial compressive stresses on the discs and their adjacent vertebral endplates. ${ }^{34}$ Once mechanical loading exceeds the bearing capacity of the vertebral endplates, microfractures may occur, further inducing the occurrence of MCs. Moreover, the alteration of mechanical loading caused by a lower PI could substantially increase the level of collagen type $\mathrm{X}$, a protein that accelerates the process of cartilage calcification, ${ }^{35}$ thus aggravating the degenerative extents by affecting the nutritional pathways of the intervertebral disc. $^{36}$ The degenerated vertebral endplates are vulnerable to damage under lesser mechanical loads and then lose their barrier function between the intervertebral disc and subchondral bone marrow, triggering "cross-talk" 37 and the initial stage of $\mathrm{MC} 1$. In the present work, PI-LL is high in all groups but pronounced in the triple-level group, probably because of a more pronounced loss of lordosis.

This study also suffers from some deficiencies. First, the present work may have inherent limitations of the retrospective study and selection bias compared to the prospective and randomized studies. Second, the small sample size, especially a relatively small number of MCs at double and triple lumbar levels, could reduce the statistical power and affect the reliability of the study findings. Additionally, we were restricted by the nature of the retrospective study from obtaining complete clinical information.

\section{Conclusions}

The present work supports the previously found connection between MCs and lumbar sagittal parameters. Further, our study identifies the MC-related characteristics, such as their types, sizes, as well as the number of involved lumbar levels by $\mathrm{MCs}$, that are significantly linked to lumbar sagittal imbalance. Overall, MC1, severe MCs, and lumbar multi-segmental MCs might contribute to lumbopelvic sagittal imbalance.

\section{Compliance with Ethics Guidelines}

Our institutional ethical committee reviewed and approved the study protocol (No. 2016-12). The present work was implemented under the guide of the principles of the Declaration of Helsinki.

\section{Acknowledgments}

We thank Dr. Qingfeng Tang for advice on statistical analysis.

\section{Author Contributions}

Xiaoping $\mathrm{Mu}$ and Jianxun Wei conceived the research topic. Jianxun wei supervised the implement of this study. 
Xiaoping $\mathrm{Mu}$ and Chengqiang $\mathrm{Yu}$ collected the data, and measured the radiographic parameters. Xiaoping $\mathrm{Mu}$ and Yufu Ou analyzed and interpreted the patient data. Xiaoping $\mathrm{Mu}$ wrote the paper. Seong Woong Kim and Karsten Schöller reviewed the draft of the manuscript. All authors made a significant contribution to the work reported, whether that is in the conception, study design, execution, acquisition of data, analysis and interpretation, or in all these areas; took part in drafting, revising or critically reviewing the article; gave final approval of the version to be published; have agreed on the journal to which the article has been submitted; and agree to be accountable for all aspects of the work.

\section{Funding}

This work was supported by the Guangxi Natural Science Foundation (2016GXNSFAA380058, 2020GXNSFAA29 7217).

\section{Disclosure}

The authors report no conflicts of interest in this work.

\section{References}

1. Deyo RA, Weinstein JN. Low back pain. N Engl J Med. 2001;344 (5):363-370. doi:10.1056/NEJM200102013440508

2. Hanımoğlu H, Çevik S, Yılmaz H, et al. Effects of Modic type 1 changes in the vertebrae on low back pain. World Neurosurg. 2019;121:e426-e432. doi:10.1016/j.wneu.2018.09.132

3. Dudli S, Liebenberg E, Magnitsky S, Lu B, Lauricella M, Lotz JC. Modic type 1 change is an autoimmune response that requires a proinflammatory milieu provided by the 'Modic disc.'. Spine $J$. 2018;18(5):831-844. doi:10.1016/j.spinee.2017.12.004

4. Hayashi T, Daubs MD, Suzuki A, et al. Motion characteristics and related factors of Modic changes in the lumbar spine. J Neurosurg Spine. 2015;22(5):511-517. doi:10.3171/2014.10.SPINE14496

5. Zhang F, Wang H, Xu H, et al. Radiological analysis on kinematical characteristics of Modic changes based on lumbar disc degeneration grade. World Neurosurg. 2018;114:e851-e856. doi:10.1016/j. wneu.2018.03.098

6. Modic MT, Steinberg PM, Ross JS, Masaryk TJ, Carter JR. Degenerative disk disease: assessment of changes in vertebral body marrow with MR imaging. Radiology. 1988;166(1):193-199. doi:10.1148/radiology.166.1.3336678

7. Modic MT, Masaryk TJ, Ross JS, Carter JR. Imaging of degenerative disk disease. Radiology. 1988;168(1):177-186. doi:10.1148/ radiology.168.1.3289089

8. Crockett MT, Kelly BS, van Baarsel S, Kavanagh EC. Modic type 1 vertebral endplate changes: injury, inflammation, or infection? AJR Am J Roentgenol. 2017;209(1):167-170. doi:10.2214/AJR.16.17403

9. Georgy MM, Vaida F, Stern M, Murphy K. Association between type 1 Modic changes and Propionibacterium acnes infection in the cervical spine: an observational study. AJNR Am J Neuroradiol. 2018;39 (9):1764-1767. doi:10.3174/ajnr.A5741

10. Lan M, Ou Y, Wang C, et al. Patients with Modic type 2 change have a severe radiographic representation in the process of lumbar degeneration: a retrospective imaging study. J Orthop Surg Res. 2019;14:298. doi:10.1186/s13018-019-1355-y
11. Dolan P, Luo J, Pollintine P, Landham PR, Stefanakis M, Adams MA. Intervertebral disc decompression following endplate damage: implications for disc degeneration depend on spinal level and age. Spine (Phila Pa 1976). 2013;38(17):1473-1481. doi:10.1097/ BRS.0b013e318290f3cc

12. van Dieën J, Weinans H, Toussaint H. Fractures of the lumbar vertebral endplate in the etiology of low back pain: a hypothesis on the causative role of spinal compression in aspecific low back pain. Med Hypotheses. 1999;53(3):246-252. doi:10.1054/mehy.1998.0754

13. Wu ZM, Ji XQ, Lian K, Liu JT. Analysis of the relationship between Modic change and spinopelvic parameters in the sagittal plane. Med Sci Monit. 2020;26:e919667. doi:10.12659/MSM.919667

14. Singh R, Yadav SK, Sood S, Yadav RK, Rohilla R. Spino-pelvic radiological parameters in normal Indian population. SICOT J. 2018;4:14. doi:10.1051/sicotj/2016003

15. Vrtovec T, Janssen MMA, Likar B, Castelein RM, Viergever MA, Pernuš F. A review of methods for evaluating the quantitative parameters of sagittal pelvic alignment. Spine J. 2012;12(5):433-446. doi:10.1016/j.spinee.2012.02.013

16. Mu X, Peng W, Yu C, et al. Modic changes of the lumbar spine - their association with the lumbar sagittal parameters: a retrospective imaging study. J Orthop Surg Res. 2020;15:220. doi:10.1186/s13018020-01745-z

17. Bland JM, Altman DG. Measuring agreement in method comparison studies. Stat Methods Med Res. 1999;8(2):135-160. doi:10.1177/ 096228029900800204

18. Chun SW, Lim CY, Kim K, Hwang J, Chung SG. The relationships between low back pain and lumbar lordosis: a systematic review and meta-analysis. Spine J. 2017;17(8):1180-1191. doi:10.1016/j. spinee.2017.04.034

19. Liow MHL, Goh GSH, Chua JL, et al. Sagittally balanced degenerative spondylolisthesis patients with increased sacral slope and greater lumbar lordosis experience less back pain after short-segment lumbar fusion surgery. Clin Spine Surg. 2019;33(5):231-235.

20. Choi S, Lee M, Kwon B. A study on difference and importance of sacral slope and pelvic sacral angle that affect lumbar curvature. Technol Health Care. 2014;22(3):467-472. doi:10.3233/THC-140805

21. Celestre PC, Dimar JR, Glassman SD. Spinopelvic parameters: lumbar lordosis, pelvic incidence, pelvic Tilt, and sacral slope what does a spine surgeon need to know to plan a lumbar deformity correction? Neurosurg Clin N Am. 2018;29(3):323-329. doi:10.1016/j.nec.2018.03.003

22. Yang X, Kong Q, Song Y, Liu L, Zeng J, Xing R. The characteristics of spinopelvic sagittal alignment in patients with lumbar disc degenerative diseases. Eur Spine J. 2014;23(3):569-575. doi:10.1007/ s00586-013-3067-z

23. Suzuki H, Imai N, Nozaki A, Hirano Y, Endo N. Anatomical sacral slope, a new pelvic parameter, is associated with lumbar lordosis and pelvic incidence in healthy Japanese women: a retrospective cross-sectional study. J Orthop Surg. 2020;28:2309499019888809. doi:10.1177/2309499019888809

24. Schwab FJ, Blondel B, Bess S, et al. Radiographical spinopelvic parameters and disability in the setting of adult spinal deformity: a prospective multicenter analysis. Spine (Phila Pa 1976). 2013;38:803-812. doi:10.1097/BRS.0b013e318292b7b9

25. Adams MA, Freeman BJC, Morrison HP, Nelson IW, Dolan P. Mechanical initiation of intervertebral disc degeneration. Spine (Phila Pa 1976). 2000;25(13):1625-1636. doi:10.1097/00007632200007010-00005

26. Xia W, Liu C, Duan S, et al. The influence of spinal-pelvic parameters on the prevalence of endplate Modic changes in degenerative thoracolumbar/lumbar kyphosis patients. PLoS One. 2018;13(5): e0197470. doi:10.1371/journal.pone.0197470

27. Nizard RS, Wybier M, Laredo J-D. Radiologic assessment of lumbar intervertebral instability and degenerative spondylolisthesis. Radiol Clin North Am. 2001;39(1):55-71. doi:10.1016/S0033-8389(05) 70263-3 
28. Wu HL, Ding WY, Shen Y, et al. Prevalence of vertebral endplate Modic changes in degenerative lumbar scoliosis and its associated factors analysis. Spine (Phila Pa 1976). 2012;37(23):1958-1964. doi:10.1097/BRS.0b013e31825bfb85

29. Kim J, Hwang JY, Oh JK, et al. The association between whole body sagittal balance and risk of falls among elderly patients seeking treatment for back pain. Bone Jt Res. 2017;6(5):337-344. doi:10.1302/2046-3758.65.BJR-2016-0271.R2

30. Wu JP, Bin WQ, Feng XJ, Qiang-Wang CMH, Cheng M-H. Relationship between Modic changes and sagittal balance parameters in the cervical spine. Med Sci Monit. 2018;24:6102-6106. doi:10.12659/MSM.909773

31. Zehra U, Cheung JPY, Bow C, et al. Spinopelvic alignment predicts disc calcification, displacement, and Modic changes: evidence of an evolutionary etiology for clinically-relevant spinal phenotypes. JOR Spine. 2020;3:e1083. doi:10.1002/jsp2.1083

32. Chen Y, Bao J, Yan Q, Wu C, Yang H, Zou J. Distribution of Modic changes in patients with low back pain and its related factors. Eur J Med Res. 2019;24:34. doi:10.1186/s40001-019-0393-6
33. Kerttula L, Luoma K, Vehmas T, Grönblad M, Kääpa E. Modic type I change may predict rapid progressive, deforming disc degeneration: a prospective 1-year follow-up study. Eur Spine J. 2012;21:1135-1142. doi:10.1007/s00586-012-2147-9

34. Strube P, Pumberger M, Sonnow L, et al. Association between lumbar spinal degeneration and anatomic pelvic parameters. Clin Spine Surg. 2018;31(6):263-267. doi:10.1097/BSD.0000000000000660

35. Roberts S, Bains MA, Kwan A, Menage J, Eisenstein SM. Type $\mathrm{X}$ collagen in the human invertebral disc: an indication of repair or remodelling? Histochem J. 1998;30:89-95. doi:10.1023/ A:1003278915981

36. Roberts S, Urban JP, Evans H, Eisenstein SM. Transport properties of the human cartilage endplate in relation to its composition and calcification. Spine (Phila Pa 1976). 1996;21:415-420. doi:10.1097/ 00007632-199602150-00003

37. Dudli S, Sing D, Hu SS, et al. Intervertebral disc/bone marrow cross-talk with Modic changes. Eur Spine J. 2017;26:1362-1373. doi:10.1007/s00586-017-4955-4

\section{Publish your work in this journal}

The Journal of Pain Research is an international, peer reviewed, open access, online journal that welcomes laboratory and clinical findings in the fields of pain research and the prevention and management of pain. Original research, reviews, symposium reports, hypothesis formation and commentaries are all considered for publication. The manuscript management system is completely online and includes a very quick and fair peer-review system, which is all easy to use. Visit http:// www.dovepress.com/testimonials.php to read real quotes from published authors. 